JÚlia Kefalás Troncon ${ }^{1}$

Dácio Leonel de Quadros Netto ${ }^{1}$

Patrícia Moretti Rehder ${ }^{1}$

José Guilherme CeCATtI ${ }^{2}$

FERNANDa Garanhani SurIta ${ }^{2}$

\section{Artigo Original}

Palavras-chave

Mortalidade materna Gravidez de alto risco/epidemiologia Bem-estar materno Complicações infecciosas na gravidez Brasil

Keywords

Maternal mortality/epidemiology Pregnancy, high risk Maternal welfare

Pregnancy complications, infectious

Brazil

\title{
Mortalidade materna em um centro de referência do Sudeste Brasileiro
}

\author{
Maternal mortality in a reference center in \\ the Brazilian Southeast
}

\section{Resumo}

OBJETIVO: Descrever a frequência da mortalidade materna em um hospital de atendimento terciário e avaliar a sua evitabilidade. MÉTODOS: $\bigcirc$ presente estudo, mediante a análise das mortes maternas ocorridas no período de 1999 a 2010 em uma maternidade de referência de Campinas - Brasil, aborda alguns dos fatores associados, as principais causas de óbito e alguns problemas de estrutura dos serviços de saúde. É um estudo descritivo retrospectivo com avaliação de variáveis sociodemográficas, história clínica e obstétrica das mulheres, além das causas do óbito. RESULTADOS: A maioria dos óbitos maternos ocorreu por causas obstétricas diretas (45\%) e evitáveis (36\%), em mulheres com gestação pré-termo que tiveram o parto por cesárea $(56 \%)$ e vários procedimentos de manejo, incluindo transfusão sanguínea, admissão em UTI e necessidade de laparotomia e/ou histerectomia. A transferência de outro hospital esteve associada ao predomínio de causas obstétricas diretas ( 19 versus $6, p=0,02$ ) e evitáveis (22 versus 9 , $p=0,01$ l. CONCLUSÃO: A mortalidade materna por causas infecciosas e hipertensivas ainda predomina. Observamos o aumento de causas clínico-cirúrgicas e neoplásicas como causa do óbito em mulheres durante o ciclo grávido puerperal.

\section{Abstract}

PURPOSE: To describe the prevalence of maternal mortality at a tertiary care hospital and to assessits preventability. METHODS: This study, through the analysis of maternal deaths that occurred during the period from 1999 to 2010 at a reference in Campinas - Brazil, CAISM/ UNICAMP, discusses some of the factors associated with the main causes of death and some structural problems of structure of the health services. It is a retrospective descriptive study with evaluation of sociodemographic variables and the medical and obstetric history of women, and the causes of death. RESULTS: The majority of maternal deaths occurred due to direct obstetric (45\%) and avoidable (36\%) causes, in women with preterm gestation, who delivered by cesarean section (56\%) and received various management procedures, including blood transfusion, ICU admission and need for laparotomy and/or hysterectomy. The hospital transfer was associated with the predominance of direct obstetric (19 versus $6, p=0.02$ ) and avoidable causes (22 versus 9 , $\mathrm{p}=0.011$. CONCLUSIONS: We conclude that, despite current advances in Obstetrics, infections and hypertensive disorders are still the predominant causes of maternal mortality. We observed an increase of clinical-surgical conditions and neoplasms as causes of death among women during pregnancy.

Correspondêncio

Fernanda Garanhani Surito Rua Alexander Fleming, 101 Cidade Universitória Prof. Zeferino Vaz

Caixa Postal: 6081

(EP: $13084-881$

Campinas (SP), Brasil

Recebido

10/02/2013

Aceito com modificacōes $12 / 08 / 2013$
Trabalho realizado na Divisão de Obstetrícia do Hospital da Mulher Prof. Dr. José Aristodemo Pinotti, Centro de Atenção Integral à Saúde da Mulher (CAISM) da Universidade Estadual de Campinas - UNICAMP - Campinas (SP), Brasil.

'Hospital da Mulher Prof. Dr. José Aristodemo Pinotti, Centro de Atenção Integral à Saúde da Mulher - CAISM - Universidade Estadual de Campinas - UNICAMP - Campinas (SP), Brasil.

2Departamento de Tocoginecologia da Faculdade de Ciências Médicas, Universidade Estadual de Campinas - UNICAMP Campinas (SP), Brasil.

Conflito de interesses: não há 


\section{Introdução}

Nos dias atuais, a saúde materna é tema de maior atenção das entidades de saúde pública do que no passado, sendo considerado um importante indicador de desenvolvimento de uma região ou país. Por isso, faz parte das Metas de Desenvolvimento do Milênio, cujo objetivo é reduzir a razão de mortalidade materna (RMM) em três quartos até $2015^{1}$. Para que essa redução da mortalidade materna ocorra, o primeiro passo é conhecer as causas de morte, entendendo melhor as necessidades e deficiências que levam aos maus resultados obstétricos.

A subnotificação da mortalidade materna e o fato de centros terciários serem receptores de uma grande área de transferência de casos, devendo estar aptos para lidar com as necessidades de uma população muito diversificada, levam a múltiplas dificuldades para reduzir a razão de mortalidade materna.

Em um estudo de desfechos perinatais adversos e near miss materno em um centro terciário em Campinas (SP), constatou-se que $48 \%$ dos casos eram transferidos de outros serviços, o que caracteriza um volume que exige esforços consideráveis para o manejo dessas situações ${ }^{2,3}$. Neste mesmo estudo, concluiu-se que menos desfechos adversos poderiam ser esperados se houvesse protocolos de conduta bem estabelecidos pelo serviço que atendeu inicialmente a gestante e, também, no serviço de referência. As principais causas de maus resultados obstétricos na região considerada foram complicações hipertensivas e hemorragias puerperais ${ }^{2}$.

No Brasil, segundo dados da Organização Mundial da Saúde (OMS), houve uma redução da RMM que é calculada dividindo-se o número de óbitos maternos pelo número de nascidos vivos, e multiplicando-se o resultado por 100.000. Em 1995, a RMM calculada para o país foi de 98/100.000NV e, em 2008, de 58/100.000NV ${ }^{1,4}$. Deve-se, entretanto, considerar a heterogeneidade do país, e a significativa subnotificação de óbitos maternos existente em muitas áreas. Curiosamente, de modo geral, nas regiões em que há melhor notificação e melhor atendimento à gestante e suas complicações, a RMM informada é maior. Já as áreas que menos notificam são as menos desenvolvidas e, portanto, aparecem com menores RMM. Isso, porque uma parcela dessas mortes não é notificada ou é totalmente desconhecida, o que faz da morte materna um problema de mais difícil reconhecimento e, consequentemente, estudo e resolução ${ }^{5}$. De qualquer maneira, a velocidade média anual de queda de mortalidade materna está aquém do esperado para que se possam atingir as metas do milênio ${ }^{3}$.

Ainda hoje, de acordo com a OMS, o risco de uma mulher morrer de causas associadas ao ciclo gravídico puerperal em um país em desenvolvimento é 36 vezes maior do que em um país desenvolvido. Para dimensionar o problema, só no ano de 2008, 358.000 mortes maternas foram registradas no mundo ${ }^{6}$. Em 2010, a OMS estima que ocorreram 287.000 óbitos maternos, com uma RMM para 210 por 100.000 nascidos vivos no mundo ${ }^{7}$.

$\mathrm{O}$ viés gerado pelas subnotificações de morte materna na interpretação dos índices ao longo do tempo foi devido à melhora dos registros após a criação de diversos comitês de mortalidade materna no país, e assim os óbitos passaram a ser mais rigorosamente notificados, mascarando a redução na razão de mortalidade materna real em relação à dos dias atuais. No passado morriam mais mulheres, porém pelo sub-registro, a razão de mortalidade materna estava subestimada ${ }^{8}$.

Pensando em uma maneira de melhor avaliar a assistência a essas mulheres que morrem e de ter uma compreensão mais global do problema, com um maior número de casos avaliados, desenvolveu-se o conceito de near miss materno. Embora este não seja o foco do presente estudo, é grande sua atual importância. Os casos de near miss materno abrangem mulheres durante a gestação, parto e puerpério, que tiveram complicações graves que quase resultaram em óbito, mas sobreviveram. Outro conceito importante é o das demoras que envolvem fatores evitáveis na assistência e que são os mesmos para os casos de near miss e morte materna. Tais fatores englobam desde a infraestrutura dos serviços e capacitação de profissionais, até o atraso da família e da própria mulher em buscar auxílio médico. Nessa condição, tal avaliação será mais facilmente realizada nos casos de near miss, já que o depoimento da mulher que sobreviveu é possível ${ }^{8,9}$. O objetivo deste estudo foi avaliar as causas da morte materna em um centro de referência terciário.

\section{Métodos}

Este é um estudo descritivo retrospectivo com o objetivo de analisar todos os casos de mortes maternas ocorridas no CAISM/UNICAMP no período de 1999 a 2010. Foram colhidos dados a partir das cópias das notificações de óbito enviadas para o Comitê de Mortalidade Materna da Regional de Campinas, e do caso, que se encontra arquivado na Comissão de Mortalidade Materna do CAISM, constituída desde 1999 e que analisa e classifica todos os óbitos maternos ocorridos.

O hospital é referência regional de complexidade na assistência à saúde da mulher e do recém-nascido. O hospital possui Unidade de Terapia Intensiva (UTI), inaugurada em 2002, com sete leitos. Dispõe de 44 leitos para obstetrícia, sendo 24 para puérperas em alojamento conjunto e 20 leitos de enfermaria para patologias obstétricas. UTI neonatal com 30 leitos, sendo 15 leitos de UTI e 15 leitos de semi-intensivo. Além de um centro obstétrico funcionando em sistema de PPP (sala de pré-parto, 
parto e puérperio) com presença de acompanhante, e salas cirúrgicas com número médio de 136 partos vaginais e 121 cesarianas por mês, sendo a taxa média de cesarianas de $47 \%$.

A taxa de ocupação em 2010 foi de $82 \%$ para os leitos de obstetrícia e de 62,4\% para UTI, o que denota a grande demanda do serviço.

São referenciadas para este hospital unidades de saúde e hospitais primários e secundários de nove principais cidades da região e de Campinas. Possui ambulatório de alto risco e especializado, um pronto atendimento que atende pacientes referenciadas e de procura espontânea. A maioria dos casos de óbito materno deste serviço são casos transferidos de outros hospitais.

As variáveis analisadas foram: idade materna, cor da pele, profissão da mulher, estado civil, antecedentes obstétricos, comorbidades associadas, uso de tabaco, álcool ou drogas, local e número de consultas no pré-natal, intercorrências do pré-natal, uso de medicações, motivo da internação, forma de término da gestação, idade gestacional ao nascimento. Também foram obtidos dados quanto à necessidade de abordagem cirúrgica, de transfusão sanguínea, anestesia utilizada, internação em UTI, presença de complicações no puerpério e período do ciclo gravídico puerperal em que ocorreu o óbito.

Foram ainda avaliadas as causas de óbito, se exame de necropsia foi realizado, se houve transferência de outro serviço, e como foi classificado o óbito após avaliação pelo Comitê de Mortalidade Materna (causas obstétricas ou não, e sua evitabilidade). Foi também analisado se o óbito havia sido classificado como materno ou não, na declaração de óbito. Para isso, foi criada uma ficha pré-codificada para a inserção dos dados, de onde os mesmos foram transcritos pelos investigadores para um banco de dados em Excel.

Análises parciais de frequência e prevalência de algumas variáveis foram realizadas e, posteriormente, feita a dicotomização do banco de dados para comparação entre as mulheres, conforme a transferência ou não de outro serviço antes de sua admissão ao hospital. Para a comparação dos grupos foram utilizados os testes de $\chi^{2}$ e exato de Fisher. O nível de significância assumido foi de $5 \%$ e o software utilizado para análise foi o SAS versão 9.02. O presente estudo foi aprovado pelo Comitê de Ética em Pesquisa da Faculdade de Ciências Médicas da Unicamp (número do protocolo 665/2011).

\section{Resultados}

No período de 12 anos, de 1999 a 2010, ocorreram 69 óbitos maternos no CAISM/UNICAMP.

Dentre os óbitos, 10 ocorreram entre mulheres adolescentes e, cerca de $32 \%$, em primigestas. Com relação às condições patológicas associadas, 25 apresentavam algum tipo de hipertensão arterial, sendo que 15 eram hipertensas crônicas e 10 tinham hipertensão gestacional ou pré-eclâmpsia. Diabetes pré-gestacional esteve presente em quatro mulheres e mais 12 desenvolveram diabetes durante a gestação. Outras sete eram cardiopatas, três eram soropositivas para o vírus HIV e oito já haviam apresentado complicações em gestações anteriores. A idade das mulheres e seus antecedentes obstétricos estão especificados na Tabela 1.

Tabela 1. Características sociodemográficas, antecedentes, via de parto, alguns critérios de manejo e transferência hospitalar dos casos de mortalidade materna do centro terciário

\begin{tabular}{|c|c|c|}
\hline Características & n & $\%$ \\
\hline \multicolumn{3}{|l|}{ Idade (anos) } \\
\hline Até 19 & 10 & 14,5 \\
\hline $20-29$ & 27 & 39,1 \\
\hline $30-39$ & 27 & 39,1 \\
\hline 40 ou mais & 5 & 7,2 \\
\hline \multicolumn{3}{|l|}{ Raça } \\
\hline Branca & 21 & 30,4 \\
\hline Não Branca & 9 & 13,0 \\
\hline Ignorado & 39 & 56,5 \\
\hline \multicolumn{3}{|l|}{ Estado civil } \\
\hline Sem parceiro fixo & 6 & 7,2 \\
\hline Com parceiro fixo & 20 & 28,9 \\
\hline Ignorado & 43 & 62,3 \\
\hline \multicolumn{3}{|l|}{ Gestações anteriores } \\
\hline 0 & 22 & 31,8 \\
\hline $1-3$ & 35 & 50,7 \\
\hline 4 ou mais & 7 & 10,1 \\
\hline Ignorado & 5 & 7,2 \\
\hline Antecedente de cesárea & 18 & 28,0 \\
\hline Antecedente de aborto & 9 & 13,9 \\
\hline \multicolumn{3}{|l|}{$\mathbf{N}^{\circ}$ de consultas de pré-natal } \\
\hline Nenhuma & 5 & 7,2 \\
\hline $1-5$ & 26 & 37,6 \\
\hline 6 ou mais & 9 & 13,0 \\
\hline Ignorado & 29 & 42,0 \\
\hline \multicolumn{3}{|l|}{ Resolução da gestação } \\
\hline Parto vaginal & 19 & 27,5 \\
\hline Cesárea & 39 & 56,5 \\
\hline Aborto & 8 & 11,5 \\
\hline MM com feto intraútero & 3 & 4,3 \\
\hline \multicolumn{3}{|l|}{ IG na resolução da gestação } \\
\hline Até 20 semanas & 8 & 11,5 \\
\hline $20-27$ semanas & 10 & 14,4 \\
\hline 28-36 semanas & 30 & 43,8 \\
\hline 37 semanas ou mais & 15 & 21,7 \\
\hline Ignorado & 6 & 8,6 \\
\hline Necessidade de transfusão & 27 & 39,1 \\
\hline Internação em UTI & 57 & 82,6 \\
\hline Necessidade de histerectomia/laparotomia & 23 & 33,3 \\
\hline Transferência de outro hospital & 40 & 57,9 \\
\hline Total & 69 & 100,0 \\
\hline
\end{tabular}

IG: idade gestacional; MM: mortalidade materna; UTI: Unidade de Terapia Intensiva. 
Com relação à forma de término da gravidez, a maior parte das mulheres teve parto entre 28 e 36 semanas, e a via de parto na maioria dos casos foi cesariana, realizada em mais da metade desses casos (Tabela 1). Com relação à assistência prestada às mulheres, pode-se avaliar a necessidade de procedimentos de manejo, incluindo a transfusão sanguínea realizada em quase $40 \%$ das mulheres, procedimentos cirúrgicos complementares como laparotomia e histerectomia realizados em mais de $30 \%$ das mulheres, e ainda a possibilidade de transferência em quase $60 \%$, associada à necessidade de cuidado intensivo em cerca de $83 \%$ dos casos (10). Estas informações encontram-se na Tabela 1.

A maior parte das MM foi obstétrica direta e evitável, ocorrendo nos primeiros 42 dias de puerpério. As causas infecciosas/sepse foram mais frequentes em $24 \%$ dos casos, seguidas de $18 \%$ de complicações clínico-cirúrgicas e $10 \%$ de causas neoplásicas (Tabela 2).

$\mathrm{Na}$ Tabela 3, há a associação entre classificação e evitabilidade da MM com o fato de ter existido transferência da mulher de outro hospital para o CAISM. Foi significativamente maior a ocorrência de MM obstétrica direta e evitável entre os casos encaminhados de outros serviços de saúde do que naqueles já seguidos na instituição de referência.

Tabela 2. Classificação das mortes maternas ocorridas no centro terciário

\begin{tabular}{|c|c|c|}
\hline & n & $\%$ \\
\hline \multicolumn{3}{|c|}{ Quanto ao período do ciclo grávido-puerperal } \\
\hline Na gestação & 8 & 11,6 \\
\hline Até 42 dias pós-parto & 53 & 76,8 \\
\hline 43 dias a 1 ano pós-parto & 7 & 10,1 \\
\hline Ignorado & 1 & 1,4 \\
\hline \multicolumn{3}{|l|}{ Quanto ao tipo de MM } \\
\hline Obstétrica direta & 31 & 44,9 \\
\hline Obstétrica indireta & 19 & 27,5 \\
\hline Não obstétrica & 6 & 8,6 \\
\hline Tardia & 9 & 13 \\
\hline Ignorada & 4 & 5,7 \\
\hline \multicolumn{3}{|l|}{ Quanto à evitabilidade } \\
\hline Evitável & 25 & 36,2 \\
\hline Inevitável & 20 & 28,9 \\
\hline Inconclusivo & 24 & 34,7 \\
\hline \multicolumn{3}{|l|}{ Causa básica } \\
\hline Infecciosa (Sepse) & 24 & 34,7 \\
\hline Hipertensiva & 8 & 11,5 \\
\hline Hemorrágica & 9 & 13,0 \\
\hline Clínico-cirúrgica & 18 & 26,0 \\
\hline Neoplásica & 10 & 14,4 \\
\hline Total & 69 & 100,0 \\
\hline
\end{tabular}

MM: mortalidade materna.
Tabela 3. Relação entre evitabilidade e classificação da mortalidade materna e a transferência de outro hospital

\begin{tabular}{|c|c|c|c|c|c|}
\hline & \multicolumn{2}{|c|}{ Transferida } & \multicolumn{2}{|c|}{ Não transferida } & \multirow{2}{*}{ Total } \\
\hline & n & $\%$ & $n$ & $\%$ & \\
\hline Evitabilidade & & & & & $p=0,02^{\star}$ \\
\hline MM evitável & 19 & 47,5 & 6 & 20,7 & 25 \\
\hline MM inevitável & 7 & 17,5 & 13 & 44,8 & 20 \\
\hline Inconclusivo & 14 & 35,0 & 10 & 34,5 & 24 \\
\hline Classificação & & & & & $\mathrm{p}=0,01^{\star}$ \\
\hline MM obstétrica direta & 22 & 55,0 & 9 & 31,1 & 31 \\
\hline MM obstétrica indirefa & 6 & 15,0 & 13 & 44,8 & 19 \\
\hline MM não obstétrica & 3 & 7,5 & 3 & 10,3 & 6 \\
\hline MM tardia & 7 & 17,5 & 3 & 10,3 & 9 \\
\hline Ignorada & 2 & 5,0 & 2 & 6,8 & 4 \\
\hline Total & 40 & & 29 & & 69 \\
\hline
\end{tabular}

Teste exato de Fisher. MM: mortalidade materna.

\section{Discussão}

Os dados apresentados no presente estudo, em concordância com estudos recentes sobre o mesmo tema, mostram como causas principais relacionadas ao óbito as hipertensivas, hemorrágicas e infecciosas ${ }^{5}$ e forma de término da gestação, predominantemente por parto cesárea $^{8}$. Embora a hipertensão represente a maior causa de óbito materno no Brasil, há pelo menos uma década, nos centros e instituições com maiores recursos, a morte por hipertensão é mais facilmente evitada e, então, se destacam as causas infecciosas e obstétricas indiretas.

Quanto à classificação dos óbitos, quase metade era de causa obstétrica direta, que teoricamente seriam óbitos evitáveis, sendo que a média na literatura, para contextos brasileiros, é maior, girando em torno de $67 \%{ }^{10}$. Como mostrado, houve associação entre elevada taxa de óbitos evitáveis e a transferência hospitalar. A necessidade de transferência implica em uma gravidade maior do quadro clínico da paciente que necessita de serviços de maior complexidade para manejo, e pode também se relacionar a um atraso no início do atendimento adequado ${ }^{11}$. Estudo conduzido neste mesmo serviço, avaliando morbidade e mortalidade materna em pacientes admitidas em UTI no período de 2002 a 2007, também apresentou taxa de transferência elevada, de $35 \%{ }^{10}$, próxima à encontrada no estudo atual, o que mostra claramente o viés associado ao estudo de mortalidade materna em um centro terciário de referência, que envolve além dos eventos locais, prováveis deficiências no atendimento primário e secundário da região.

Quanto à evitabilidade, também encontramos taxas elevadas $(36,2 \%)$ dentre os óbitos analisados, da mesma forma que demonstra a literatura, quando há predomínio de causas obstétricas diretas. Tais fatos relacionam-se ao 
conceito já abordado dos atrasos na assistência, que vão desde a demora na procura pelo serviço, demora do serviço secundário em reconhecer a gravidade do caso, necessidade de transferência para centro terciário e, finalmente, as condutas no centro de referência ${ }^{9,10}$.

A questão da demora no atendimento obstétrico e sua associação com desfechos desfavoráveis são descritas desde meados dos anos $1980^{12,13}$, e seu estudo torna-se importante na medida que tenta identificar possíveis intervenções que, se realizadas em momento oportuno, poderiam modificar a evolução de um caso grave. As demoras podem ser divididas em três tipos: a primeira relacionada à mulher e seus familiares, na procura por assistência médica. A segunda é a que ocorre nos níveis primário ou secundário da assistência, pela dificuldade de acesso aos serviços, dificuldade de transporte e à demora da equipe de saúde em diagnosticar e encaminhar casos de maior risco e, dessa forma, levar a um atraso no atendimento ideal, o que nas situações mais graves pode ser decisivo para o desfecho do caso. A terceira demora é a que ocorre relacionada à qualidade da atenção na unidade de saúde em que medidas específicas, se não tomadas em momento oportuno, também podem ser a causa de desfechos não satisfatórios ${ }^{14}$. Embora o presente estudo não tenha especificamente investigado demoras, os achados potencialmente relacionados às demoras são similares a outros que abordam o mesmo tema ${ }^{15,16}$.

Nesta série de casos predominaram causas obstétricas de óbito materno, o que geralmente ocorre na análise de dados de populações de países em desenvolvimento ${ }^{17} \mathrm{e}$ que se associa a deficiências na condução de casos, com possibilidade de reversão se atendidos de forma correta no momento ideal. Um exemplo claro para esses casos é a hemorragia pós-parto, que pode ser evitada na maioria dos casos com medidas simples (profilaxia com ocitocina pós-parto), mas que quando ocorre pode ser revertida se abordada rapidamente (uterotônicos, suturas hemostáticas ou histerectomia $)^{10}$. A condução de casos de hemorragia obstétrica que envolva a necessidade de transfusão, histerectomia e internação em UTI associa-se a dificuldades no manejo. Assim, avaliando a assistência prestada a essas mulheres, depara-se com o conceito dos "critérios de manejo" que fazem parte de estratégias já bem estabelecidas para a redução da morbimortalidade materna ${ }^{10,18,19}$.

Na casuística avaliada, as causas básicas mais frequentes de MM foram as infecciosas, hipertensivas e hemorrágicas. Nota-se, entretanto, número considerável de causas clínicocirúrgicas e neoplasia como causa do óbito. Encontramos um total de dez casos de óbito associados a causas neoplásicas, o que também denota a maior complexidade de casos atendidos em um centro terciário. Chama a atenção, ainda, o diagnóstico de neoplasias em mulheres jovens, em alguns casos feito apenas durante a gestação, complicando significativamente o tratamento e a evolução da doença.

Traçando um paralelo com estudo conduzido previamente na mesma região ${ }^{20}$, observamos que $39,1 \%$ dos óbitos ocorreram na faixa etária de 20 a 29 anos, enquanto na avaliação dos óbitos ocorridos também na cidade de Campinas, de 1992 a 1994, dos 20 óbitos, oito ocorreram acima dos 30 anos. Contudo, não foi registrado na ocasião nenhum óbito acima dos 34 anos, enquanto que na presente casuística, 39,1\% ocorreram de 30 a 39 anos e 7,2\% acima dos 40 anos. Tal fato possivelmente reflete a taxa crescente de mulheres que engravidam em idade progressivamente mais avançada, devido a mudanças na realidade socioeconômica e preferências pessoais.

Segundo dados do Manual dos Comitês de Mortalidade Materna $^{21}$, no Brasil mantém-se ainda o predomínio absoluto de causas obstétricas diretas e mortes classificadas como evitáveis. Em nossa amostra obtivemos valores inferiores, denotando provavelmente características da transição epidemiológica nessa região, bem como melhor estrutura e qualidade de atenção oferecida às mulheres durante a gestação. Todavia, é importante lembrar que em todas as variáveis analisadas observamos a deficiência no registro de dados, e que isso também representa prejuízo na análise e classificação dos óbitos.

A mortalidade materna é sempre um tema desafiador, pelo impacto na sociedade, pela sua possível evitabilidade, ou ainda pela dificuldade na redução da sua ocorrência em todo o mundo. Apesar de inúmeras estratégias utilizadas, permanece como um problema de difícil solução, o que torna pouco provável a possibilidade de se atingirem as metas do milênio.

\section{Referências}

1. Hogan MC, Foreman KJ, Naghavi M, Ahn SY, Wang M, Makela SM, et al. Maternal mortality for 181 countries, 1980-2008: a systematic analysis of progress towards Millennium Development Goal 5. Lancet. 2010;375(9726): 1609-23.

2. Amaral E, Souza JP, Surita F, Luz AG, Sousa MH, Cecatti JG, et al. A population-based surveillance study on severe acute maternal morbidity (near miss) and adverse perinatal outcomes in Campinas,
Brazil: The Vigimoma Project. BMC Pregnancy Childbirth [Internet]. 2011 [cited 2013 Jan 20];1 1:9. Available from: <http://www. biomedcentral.com/1471-2393/11/9>

3. World Health Organization. Global Health Observatory (GHO) [Internet]. Maternal and reproductive health; 2010 [cited 2013 Jan 20]. Available from: <http://www.who.int/gho/maternal_health/ en/index.html> 
4. Betrán AP, Wojdyla D, Posner SF, Gülmezoglu AM. National estimates for maternal mortality: an analysis based on the WHO systematic review of maternal mortality and morbidity. BMC Pregnancy Childbirth [Internet]. 2005 [cited 2013 Jan 20];5:131. Available from: <http://www.biomedcentral.com/1471-2458/5/131>

5. Morse ML, Fonseca SC, Barbosa MD, Calil MB, Eyer FPC. Mortalidade materna no Brasil: o que mostra a produção científica nos últimos 30 anos? Cad Saúde Pública. 2011 ;27(4): 623-38.

6. Victora CG, Aquino EM, Leal MC, Monteiro CA, Barros FC, Szwarcwald CL. Maternal and child health in Brazil: progress and challenges. Lancet. $2011 ; 377(9780): 1863-76$.

7. World Health Organization. World Bank. UNICEF. United Nations Population Fund [Internet]. Trends in maternal mortality: 1990 to 2010; 2012 [cited 2013 Jan 20]. Available from: <http:// www.unfpa.org/webdav/site/global/shared/documents/ publications/2012/Trends_in_maternal_mortality_A4-1.pdf>

8. Cecatti JG, Souza JP, Parpinelli MA, Haddad SM, Camargo RS, Pacagnella RC, et al. Brazilian network for the surveillance of maternal potentially life threatening morbidity and maternal near-miss and a multidimensional evaluation of their long term consequences. Reprod Health [Internet]. 2009 [cited 2013 Jan 20];6:15. Available from: <http://www.reproductive-health-journal.com/content/6/1/15>

9. Luz AG, Tiago DB, Silva JC, Amaral E. [Severe maternal morbidity at a local reference university hospital in Campinas, São Paulo, Brazil]. Rev Bras Ginecol Obstet. 2008;30(6):281-6. Portuguese.

10. Oliveira Neto AF, Parpinelli MA, Cecatti JG, Souza JP, Sousa MH. Factors associated with maternal death in women admitted to an intensive care unit with severe maternal morbidity. Int J Gynaecol Obstet. 2009; 105(3):252-6.

11. Amaral E, Luz AG, Souza JP. [The severe maternal morbidity for the qualification of care: utopia or necessity?]. Rev Bras Ginecol Obstet. 2007;29(9):484-9. Portuguese.

12. Thaddeus $S$, Maine D. Too far to walk: maternal mortality in context. Newsl Womens Glob Netw Reprod Rights. 1991;(36):22-4.
13. Thaddeus S, Maine D. Too far to walk: maternal mortality in context. Soc Sci Med. 1994;38(8): 1091-110.

14. Pacagnella RC, Cecatti JG, Osis M, Souza JP. The role of delays in severe maternal morbidity and mortality: expanding the conceptual framework. Reprod Health Matters. 2012;20(39):155-63.

15. Issah K, Nang-Beifubah A, Opoku CF. Maternal and neonatal survival and mortality in the Upper West Region of Ghana. Int J Gynaecol Obstet. 2011;113(3):208-10.

16. Shah N, Hossain N, Shoaib R, Hussain A, Gillani R, Khan NH. Socio-demographic characteristics and the three delays of maternal mortality. J Coll Physicians Surg Pak. 2009;19(2):95-8.

17. Haddad N, Silva MB. Mortalidade feminina em idade reprodutiva no estado de São Paulo, Brasil, 1991-1995: causas básicas de óbito e mortalidade materna. Rev Saúde Pública. 2000;34(1):64-70.

18. Say L, Pattinson RC, Gülmezoglu AM. WHO systematic review of maternal morbidity and mortality: the prevalence of severe acute maternal morbidity (near miss). Reprod Health [Internet]. 2004 [cited 2013 Jan 20];1:3. Available from: <http://www. reproductive-health-journal.com/content/1/1/3>

19. Cecatti JG, Souza JP, Oliveira Neto AF, Parpinelli MA, Sousa $M H$, Say $L$, et al. Pre-validation of the WHO organ dysfunction based criteria for identification of maternal near miss. Reprod Health [Internet]. 2011 [cited 2013 Jan 20];8:22. Available from: <http://www.reproductive-health-journal.com/content/8/1/22>

20. Parpinelli MA, Faúndes A, Surita FG, Pereira BG, Cecatti JG. [Maternal mortality in Campinas, during the period 1992-1994]. Rev Bras Ginecol Obstet. 1999;21 (4):227-32. Portuguese.

21. Brasil. Ministério da Saúde. Secretaria de Atenção à Saúde. Departamento de Ações Programáticas Estratégicas [Internet]. Manual dos Comitês de Mortalidade Materna. $3^{a}$ ed. Brasília (DF): Editora do Ministério da Saúde; 2009 [citado 2013 Jan 20]. (Série A Normas e Manuais Técnicos). Disponível em: <http:// www.saude.ms.gov.br/controle/ShowFile. php?id=127236> 